\title{
Prospects of the Thermoelectricity Based on Organic Materials
}

\author{
Anatolie Casian* and Ionel Sanduleac \\ Technical University of Moldova, MD-2004, Chisinau, Republic of Moldova. \\ * acasian@mail.utm.md
}

\begin{abstract}
Thermoelectric properties of existing crystals of tetrathiotetracene-iodide, $\mathrm{TTT}_{2} \mathrm{I}_{3}$, are modeled in the most complete 3D physical model. The expected values of the thermoelectric power factor and of the thermoelectric figure of merit are determined for crystals with different degrees of perfection and carrier concentration. Optimal values of electrical conductivity, Seebeck coefficient, and electronic thermal conductivity in order to achieve the predicted values of the thermoelectric figure of merit are calculated.
\end{abstract}

\section{Introduction}

As it is known, almost $90 \%$ of world annual energy consumption (which now is around 15 terawatt-years and is expected to be doubled by 2050 [1]) is generated by heat engines that use fossil fuel combustion, oil, natural gas and coal as a heat sources. These engines usually operate at 30-40 per cent efficiency. Thus, an enormous quantity of heat is not used and is lost to the environment. It is natural that there is a significant interest in finding cost-effective technologies for generating electricity from this waste heat. It is expected that namely thermoelectric generators will serve for these goals.

Thermoelectric convertors serve also as refrigerators transforming directly the electrical energy into cold. Thermoelectric generators and refrigerators have evident advantages in comparison with the ordinary ones: no moving parts and high reliability, can be made very compacted, noiseless work. The main parameter that determines the possibility of a material to be used in the thermoelectric converters of energy is the dimensionless thermoelectric figure of merit $Z T$, where $T$ is the operating temperature. Now the largest commercially applied thermoelectric materials on the base of $\mathrm{Bi}_{2} \mathrm{Te}_{3}$ have $Z T \sim 1$ near room $T$. It is a rather low value. Therefore, now the thermoelectric convertors are used mainly in special cases, where the comfort is more important than the efficiency. A value of $Z T$ equal or bigger than 3 would make the solid-state convertors economically competitive with the ordinary used ones.

Harman has been obtained $Z T \sim 3$ in PbTeSe quantum dot superlattices [2], and even $Z T \sim 3.5[3,4]$. However, the technology to obtain such structures is complicated, expensive and can not be applied for large scale production.

In the last years it is observed an increasing effort in the search and investigation of new organic materials for the use in more efficient and low cost thermoelectric generators in order to convert even a part of low-grade waste heat into electricity.

In poly(3,4-ethylenedioxythiophene) (PEDOT) doped by poly(styrenesulphonate) (PSS) thin films a value of $Z T=0.42$ at room temperature has been measured [5]. In nanocomposites the highest value of $Z T=$ 0.57 at room temperature was obtained in phenyl acetylene-capped silicon nano particles [6]. A value of $Z T \sim 15$ at room temperature has been predicted in molecular nanowires of conducting polymers [7]. Even higher values of $Z T$ were predicted by us in highly conducting quasi-onedimensional (Q1D) charge transfer organic crystals [8]. However, these high values of $Z T$ have been predicted in strictly $1 \mathrm{D}$ 
approximation. As it has been demonstrated in [9] for a 2D physical model, the results of 1D approximation are valuable in not very pure crystals, when the carrier mobility is limited by the impurity scattering and the interchain interaction does not give important contribution. In $\mathrm{TTT}_{2} \mathrm{I}_{3}$ crystals grown from solution [10] with not very high electrical conductivity $\sigma_{x x}=1.8 \square 10^{5} \Omega^{-1} \mathrm{~m}^{-1}$ along conductive chains only $Z T \cong 0.1$ was really obtained at room temperature [11].

The aim of this paper is to present a more detailed modeling of the thermoelectric properties of $\mathrm{TTT}_{2} \mathrm{I}_{3}$ crystals in a more complete 3D physical model. The values of $Z T$ expected in this case are determined. The optimal thermoelectric parameters necessary in order to achieve increased values of $Z T$ are also determined.

\section{Crystal model}

From the structural point of view the Q1D organic crystals are formed from linear chains or stacks of molecules packed info a three-dimensional lattice. The interaction between the molecules along the chains is much bigger than in transversal direction. As a result, the mechanical properties are very anisotropic and the crystals have a needle-like form. In the $\mathrm{TTT}_{2} \mathrm{I}_{3}$ crystals the TTT and iodine chains are separated and only TTT chains are conductive. The carriers are holes. They are moving mainly along the chains where they were created and rarely jump from one chain to another. These jumps determine the kinetic interchain interaction. The physical 3D model is described in [12].

As in the 1D approximation, two electron-phonon interaction mechanisms and also the scattering of carriers on impurities are taken into account. The first mechanism is similar to that of deformation potential and is determined by the variation of the transfer energies $w_{1}, w_{2}$ and $w_{3}$ of an electron from the given molecule to the nearest ones in three directions, caused by the lattice acoustic vibrations. The three coupling constants are proportional to the derivatives $w_{1}^{\prime}, w_{2}^{\prime}$ and $w_{3}^{\prime}$ of $w_{1}, w_{2}$ and $w_{3}$ with respect to intermolecular distances. The second mechanism is polaron similar and is determined by the variation, caused by the same acoustic vibrations, of the polarization energy of molecules surrounding the conduction electron. The coupling constant of this interaction is determined by the mean polarization of TTT molecule $\alpha_{0}$.

It is important that under certain conditions, the interference between these electron-phonon interactions is possible. As a result, these two interactions significantly compensate each other for some states in the conduction band. In consequence, the relaxation time as a function of carrier energy takes a maximum for these states. The carriers in these states have increased mobilities. It is favorable for thermoelectric applications, because higher mobilities ensure higher values of $Z T$.

The impurities are considered point-like and neutral. The impurity scattering rate is described by a dimensionless parameter $D_{0}$ which is proportional to impurity concentration and can be made very small, if the crystal purity is sufficiently high.

\section{Results and discussions}

It is considered that a weak electrical field and a weak temperature gradient are applied along the conductive chains. The linearized kinetic equation takes the form of the Boltzmann one. Near room temperature the scattering processes can be considered elastic. Then the kinetic equation is solved analytically and the expressions for electrical conductivity $\sigma_{x x}$, Seebeck coefficient $S_{x x}$, thermoelectric power factor $P_{x x}$, electronic thermal conductivity $\kappa_{x x}^{e}$ and thermoelectric figure of merit $(Z T)_{x x}$ along chains can be presented through the threedimensional transport integrals $R_{\mathrm{n}}$

$$
\begin{gathered}
\sigma_{x x}=\sigma_{0} R_{0}, \\
S_{x x}=\left(k_{0} / e\right)\left(2 w_{1} / k_{0} T\right) R_{1} / R_{0},
\end{gathered}
$$




$$
\begin{gathered}
P_{x x}=\sigma_{x x} S_{x x}^{2} \\
\kappa_{x x}^{e}=4 w_{1}^{2} \sigma_{0} /\left(e^{2} T\right)\left(R_{2}-R_{1}^{2} / R_{0}\right) \\
(Z T)_{x x}=\sigma_{x x} S_{x x}^{2} T /\left(\kappa_{x x}^{L}+\kappa_{x x}^{e}\right)
\end{gathered}
$$

where:

$$
\sigma_{0}=\left(2 e^{2} M v_{s 1}^{2} w_{1}^{3} r\right) /\left(\pi^{2} \hbar a b c\left(k_{0} T\right)^{2}{w_{1}^{\prime}}^{2}\right)
$$

Here $e$ is the carrier charge, $k_{0}$ is the Boltzmann constant, $T$ is the temperature, $\kappa_{x x}^{L}$ is the lattice thermal conductivity, $M$ is the mass of TTT molecule, $v_{\mathrm{s} 1}$ is the sound velocity along chains, $r$ is the number of molecular chains through the transversal section of the elementary cell, $a, b, c$ are the lattice constants, $w_{1}^{\prime}$ is the derivative of transfer energy $w_{1}$ with respect to intermolecular distance. The expression for $R_{\mathrm{n}}$ is somewhat cumbersome. It is presented in [12].

For $\mathrm{TTT}_{2} \mathrm{I}_{3}$ the crystal parameters are: $M$ $=6.5 \square 10^{5} m_{e} \quad\left(m_{e}\right.$ is the mass of free electron), $a=18.35 \AA, b=4.96 \AA, c=18.46$ $\AA, v_{\mathrm{s} 1}=1.5 \square 10^{3} \mathrm{~m} / \mathrm{s}, w_{1}=0.16 \mathrm{eV}, w_{1}^{\prime}$ $=0.26 \mathrm{eV}^{-1}, r=4, k_{x x}^{L}=0.6 \mathrm{WK}^{-1} \mathrm{~m}^{-1}$, $w_{2} / w_{1}=w_{2}^{\prime} / w_{1}^{\prime}=d_{1}, w_{3} / w_{1}=w_{3}^{\prime} / w_{1}^{\prime}=d_{2}=$ $d_{1}=0.015$, because the lattice constants $a$ and $c$ are very close to each other. The mean polarizability of TTT molecules was taken as in [9] $\alpha_{0}=45 \AA^{-3}$.

In Fig. 1 the dependences of electrical conductivity along chains $\sigma_{x x}$ as functions of dimensionless Fermi energy $\varepsilon_{F}$ in units of $2 w_{1}$ at room temperature are presented for different values of $D_{0}$.

The curves with the lowest maximum $\left(D_{0}=0.6\right)$ correspond to crystals grown form solution [10] with $\sigma_{x x}=1.8 \square 10^{5} \Omega^{-1} \mathrm{~m}^{-1}$ in the stoichiometric compounds, $\varepsilon_{F}=0.35$. The curves with the middle maximum $\left(D_{0}=\right.$ $0.1)$ correspond to more perfect crystals grown by gas phase method [13] with stoichiometric electrical conductivity $\sigma_{x x} \sim$ $10^{6} \Omega^{-1} \mathrm{~m}^{-1}$, and the curves with the highest maximum correspond to still more perfect crystals with $\sigma_{x x} \sim 2.5 \square 10^{6} \Omega^{-1} \mathrm{~m}^{-1}$ not synthesized yet.

It is seen that in the first two groups of crystals the results in the 3D model coincide with those from the $1 \mathrm{D}$ model. In this case the carrier mobility is limited by the scattering on impurities. Only in the most perfect crystals a small diminution of $\sigma_{x x}$ in the $3 \mathrm{D}$ model is observed caused by the contribution of weak scattering of carriers on adjacent chains.

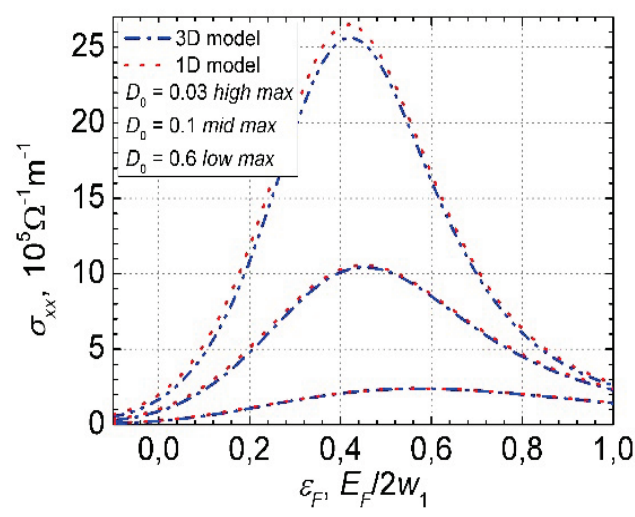

Fig.1. Electrical conductivity $\sigma_{x x}$ along chains as a function of Fermi energy $\varepsilon_{F}$.

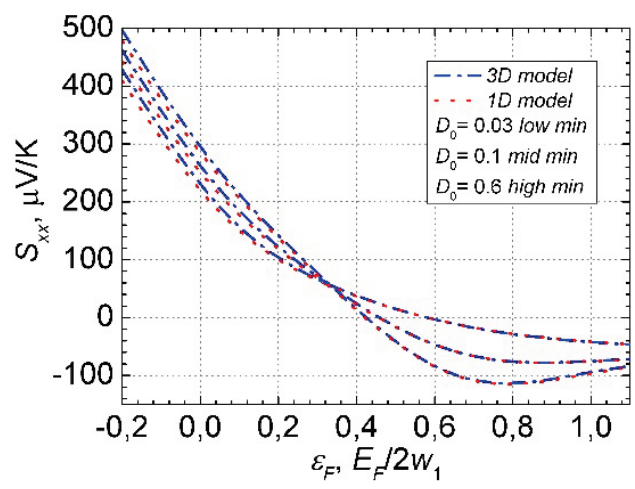

Fig.2. Seebeck coefficient $S_{\mathrm{xx}}$ along chains as a function of Fermi energy $\varepsilon_{F}$.

In Fig. 2 the dependences of Seebeck coefficient along chains $S_{x x}$ as functions of dimensionless Fermi energy $\varepsilon_{F}$ are 
presented. It is seen that so as $S_{x x}$ is the ratio of transport integrals (see (2)), it is less sensitive to the carrier scattering on adjacent chains and therefore the results of 3D and 1D models are very close in the whole interval of $\varepsilon_{F}$ variation. For stoichiometric compounds $\left(\varepsilon_{F}=0.35\right) S_{x x}$ weakly depends on crystal purity (parameter $D_{0}$ ) and takes values between 45 and $40 \mu \mathrm{V} / \mathrm{K}$, near to those that are observed experimentally. But with the decrease of $\varepsilon_{F}, S_{x x}$ grows considerably and for $\varepsilon_{F}=0.2$ achieves values $\sim 100-150$ $\mu \mathrm{V} / \mathrm{K}$, or even $\sim 300 \mu \mathrm{V} / \mathrm{K}$ for $\varepsilon_{F}=0$.

The thermoelectric power factor $P_{x x}$ (2) is an important parameter which determines the possibility of the given material to be used as sensitive element in the thermoelectric detectors of infrared radiation and in systems of night vision. Values as high possible are needed. The dependences of power factor along chains $P_{x x}$ as functions of Fermi energy $\varepsilon_{F}$ are presented in the Fig. 3. It is seen that for not perfect crystals grown from solution $P_{x x}$ obtains small values in the whole interval of $\varepsilon_{F}$ variation. In more perfect crystals grown by gas phase method $\left(D_{0}=0.1\right)$ the maximum of $P_{x x}$ is equal to $79 \square 10^{-4} \mathrm{Wm}^{-1} \mathrm{~K}^{-}$ ${ }^{1}$, or almost two times higher than in $\mathrm{Bi}_{2} \mathrm{Te}_{3}$. But in the most perfect crystals $\left(D_{0}=0.03\right)$ $P_{x x}$ is almost six time higher than in $\mathrm{Bi}_{2} \mathrm{Te}_{3}$.

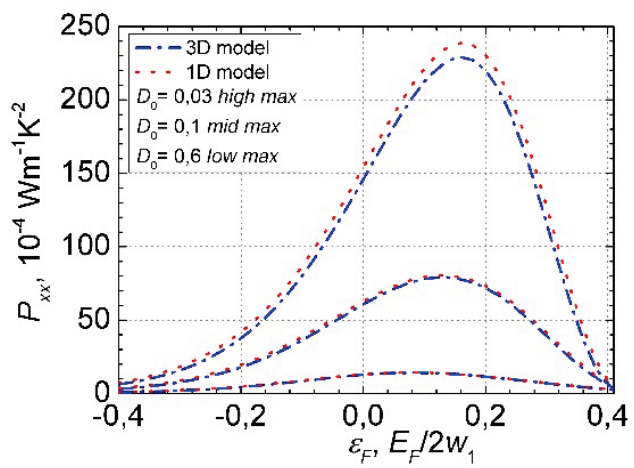

Fig.3. Thermoelectric power factor $P_{x x}$ along chains as a function of Fermi energy $\varepsilon_{F}$.

The dependences of electronic thermal conductivity along chains $\kappa_{x x}^{e}$ as functions of Fermi energy $\varepsilon_{F}$ are presented in the Fig. 4. It is seen that for given degree of crystal perfection the deviations of results for the 3D model are negligible with respect to those of 1D model. But with the increase of crystal perfection the electronic thermal conductivity $\kappa_{x x}^{e}$ grows considerable.

However, the maximums of $\kappa_{x x}^{e}$ are displaced to higher values of $\varepsilon_{F}$ with respect to those of $\sigma_{x x}$. It is very important, because it leads to violation of the WiedemannFranz law and to diminution of the Lorenz number in the intervals of $\varepsilon_{F}$ variation, where an increase of $(Z T)_{x x}$ is expected.

The dependences of the thermoelectric figure of merit $(Z T)_{x x}$ along chains as a function of Fermi energy $\varepsilon_{F}$ at room temperature are presented in Fig. 5.

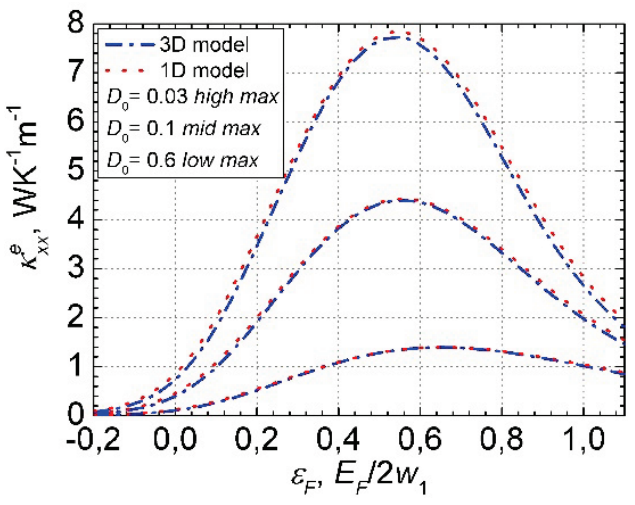

Fig.4. Electronic thermal conductivity $\kappa_{x x}^{e}$ as a function of Fermi energy $\varepsilon_{F}$.

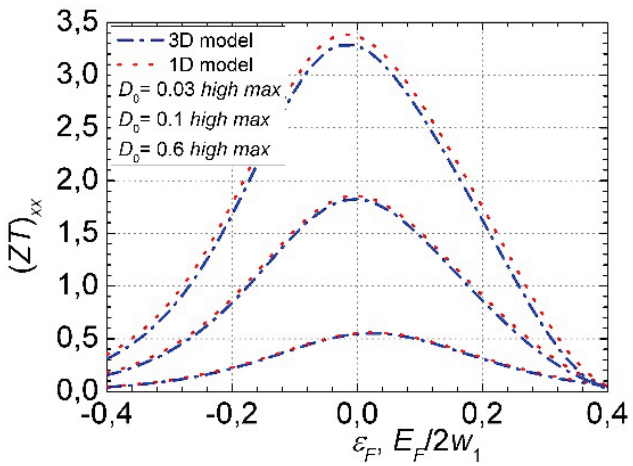

Fig.5. Thermoelectric figure of merit $(Z T)_{x x}$ along chains as a function of Fermi energy $\varepsilon_{F}$. 
It is seen the in stoichiometric crystals $(Z T)_{x x}$ is very small $\sim 0.1$ in all crystals presented here and practically does not depend on the degree of crystal perfection. But with the decrease of $\varepsilon_{F}$, or of carrier concentration $(Z T)_{x x}$ grows. In existing crystals with stoichiometric electrical conductivity $\sigma_{x x} \sim 10^{6} \Omega^{-1} \mathrm{~m}^{-1}$ (the curves with the middle maximum, $D_{0}=0.1$ ) the maximum of $(Z T)_{x x}$ is predicted to achieve a value of 1.7. The expected crystal parameters in this case are: $\sigma_{x x}=8.8 \times 10^{4} \Omega^{-}$ ${ }^{1} \mathrm{~m}^{-1}, S_{x x}=262 \mu \mathrm{V} / \mathrm{K}, \kappa_{x x}^{e}=0.40 \mathrm{WK}^{-1} \mathrm{~m}^{-1}$.

In still more perfect crystals with stoichiometric $\sigma_{x x} \sim 2.5 \times 10^{6} \Omega^{-1} \mathrm{~m}^{-1}$ not synthesized yet (the curves with the highest maximum, $D_{0}=0.03$ ) the maximum of $(Z T)_{x x}$ is predicted to be even higher than 3 . The expected crystal parameters in this case are: $\sigma_{x x}=1.7 \times 10^{5} \Omega^{-1} \mathrm{~m}^{-1}, S_{x x}=295 \mu \mathrm{V} / \mathrm{K}$, $\kappa_{x x}^{e}=0.73 \mathrm{WK}^{-1} \mathrm{~m}^{-1}$, very good results. It is important to note that the main contribution to the increase of $(Z T)_{x x}$ comes from the increase of the power factor $P_{x x}=\sigma_{x x} S_{x x}{ }^{2}$. Thus, in the last case $P_{x x}=1.5 \times 10^{-2} \mathrm{Wm}^{-1} \mathrm{~K}^{-}$ ${ }^{2}$, or almost four times higher than in $\mathrm{Bi}_{2} \mathrm{Te}_{3}$. Still higher values of $(Z T)_{x x}$ are predicted in more perfect crystals [14].

Of case, in order to achieve these values of $(Z T)_{x x}$ it is necessary to diminish considerably the carrier concentration. It is possible, because the $\mathrm{TTT}_{2} \mathrm{I}_{3}$ crystals admit non stoichiometric composition with excess or deficiency of iodine. The latter plays the role of acceptors and determines the carrier concentration.

\section{Conclusions}

It is demonstrated that the highly conductive quasi-one-dimensional crystals of tetrathiotetracene-iodide, $\mathrm{TTT}_{2} \mathrm{I}_{3}$, are very prospect for thermoelectric applications. Modeling of their thermoelectric properties has shown that after the respective increase of crystal perfection and optimization of crystal parameters, values of the thermoelectric power factor $P_{x x}=1.5 \square 10^{-2} \mathrm{Wm}^{-1} \mathrm{~K}^{-2}$ are predicted, or of almost six times higher than in $\mathrm{Bi}_{2} \mathrm{Te}_{3}$. The thermoelectric figure of merit $(Z T)_{x x}$ is expected to be higher than 3 , of more than three times higher than in the best $\mathrm{Bi}_{2} \mathrm{Te}_{3}$, the largest commercially applied thermoelectric material.

\section{Acknowledgment}

This work was partly supported by EU Commission FP7 program (Grant No. 308768 and by Academy of Sciences of Moldova scientific program (the project No. 14.02.116F).

\section{References}

27) A. Slaoui, R. T. Collins. MRS Bulletin, 32, 211, 2007.

28) M. S. Dresselhaus and J. P. in: Thermoelectric Handbook, Macro to Nano, Ed. by D. M. Rowe, CRC Press, 2006, Chap. 39 (and references therein).

29) C. B. Vining, Proc. of $5^{\text {th }}$ Europe Conf. on Thermoel., Odessa. 2007, p. 5-10.

30) T. C. Harman, M. P. Walsh, B. E. LaForge, G. W. Turner. J. of Electronic Mater. v. 34, pp. L19-L22, 2005.

31) G-H. Kim, L. Shao, K. Zhang and K. P. Pipe. Nat. Mater. Vol. 12, 719, 2013, DOI: 10.1038/NMAT3635.

32) S. P. Ashby, J. García-Cañadas, G. Min \& Y. Chao, JEM, 42, 1495 (2013).

33) Y. Wang, J. Zhou, and R. Yang. J. Phys. Chem. C, 115, 24418 (2011).

34) A. Casian in: Thermoelectric Handbook, Macro to Nano, Ed. by D. M. Rowe, CRC Press, 2006, Chap.36.

35) I. I. Sanduleac, A. I. Casian, J. Pflaum, $J$ of Nanoelectronics and Optoelectronics, 9, 247-252, 2014.

36) V.F. Kaminskii, M.L. Khidekel', R.B. Lyubovskii et all. Phys. Status Solidi A 44, 77 (1977).

37) A. Casian, I. Sanduleac. J. Electron. Mat., 43, 3740, 2014.

38) I. I. Sanduleac, J. of Thermoelectricity, No 4, 50, 2014.

39) B. Hilti and C.W. Mayer. Helvetica Chimica Acta, 61, Nr 40, 501, (1978).

40) A. I. Casian, J. Pflaum, I. I. Sanduleac. $J$. of Thermoelectricity, 2015, in press. 